\section{Intraocular metastases of cutaneous malignant melanoma: a case report and review of the literature}

\begin{abstract}
Cutaneous malignant melanoma metastatic to the eye is rare but has been documented. The usual presentation is after the diagnosis of disseminated metastases. A 40-year-old Caucasian man presented with floaters and hazy vision due to ocular metastasis. He developed painful intractable rubeotic glaucoma leading to enucleation.

Histopathological examination confirmed anterior segment tumour adherent to the iris that was consistent histopathologically with the primary cutaneous melanoma.
\end{abstract}

Key words Cutaneous malignant melanoma, Intraocular, Metastases, Rubeotic glaucoma

Metastatic melanoma to the eye is considered to be rare. The distiction between metastatic and primary melanoma is important because the prognosis and methods of treatment differ. Metastatic cutaneous melanoma generally indicates a grave outcome. ${ }^{1}$ We report a patient with a history of cutaneous melanoma presenting with anterior segment metastasis who subsequently developed rubeotic glaucoma.

\section{Case report}

A 40-year-old Caucasian farmer presented with floaters and hazy vision of a few weeks' duration. Visual acuity at presentation was $6 / 12$ right and 6/6 left. Marked anterior chamber cells and flare, a few mutton fat keratic precipitates and rubeosis iridis were noted. The intraocular pressure was normal at presentation. The right iris appeared pushed forward at the 9 o'clock position and gonioscopy after dilation revealed a grey mass at the ciliary body (Fig. 1). Fundal examination was unremarkable. A malignant melanoma had been removed from his back 2 years prior to ophthalmic presentation and axillary secondaries removed a year later. Systemic investigation revealed no further evidence of metastasis. A presumptive diagnosis of metastatic melanoma to the ciliary body was made. A month after ophthalmic presentation the intraocular pressure rose to $40 \mathrm{mmHg}$ with evidence of rubeosis. Tumour regression did not occur with external beam radiation (total radiation $1000 \mathrm{~Gy}$ over three fractions). In view of the intractable pain and poor vision the right eye was enucleated. Four months after enucleation the patient died of cerebral metastases.

Gross pathological examination of the globe $(24 \times 25 \times 23 \mathrm{~mm})$ showed a white dumb-bell shaped tumour in the posterior chamber measuring $5 \mathrm{~mm}$ in diameter attached to the iris and adherent to the lens (Fig. 2).

Microscopy confirmed tumour attached to the iris extending in an irregular sheet across the ciliary body (Fig. 3) but sparing the retina. Tumour cells were also seen in the anterior chamber drainage angle. The tumour deposits were composed of polygonal cells with ovoid nuclei and prominent nucleoli. There were widespread mitotic figures and multifocal necrosis. Melanin pigmentation was scanty. No associated naevoid component was identified. Melanin stains were focally positive and immunocytochemistry demonstrated a positive staining reaction with antibodies to $\mathrm{S} 100$ and neuron-specific enolase, with patchy weak reaction to HMB-45. A histological diagnosis of intraocular metastatic melanoma from a cutaneous primary was made.

\section{Comment}

Differentiation between metastatic melanoma and primary melanoma of the eye has significant clinical consequences. A high index of suspicion is essential when approaching such cases as the patient may not volunteer information relating to the previous excision of pigmented cutaneous lesions. Cutaneous melanoma presenting for the first time with intraocular metastases is rare. However, Font et al. ${ }^{2}$ reported two cases in which presenting features were hyphaema, therapy-resistant
K. Ramaesh Princess Alexandra Eye Pavilion

Chalmers Street Edinburgh EH3 9HA, UK

K. Ramaesh

J.W.V. Marshall

B. Dhillon

Princess Alexandra Eye Pavilion

Edinburgh, UK

S.B. Wharton

Neuropathology Laboratory Department of Pathology Western General Hospital Edinburgh, UK Accepted in revised form: 9 December 1998
Received: 12 June 1998 


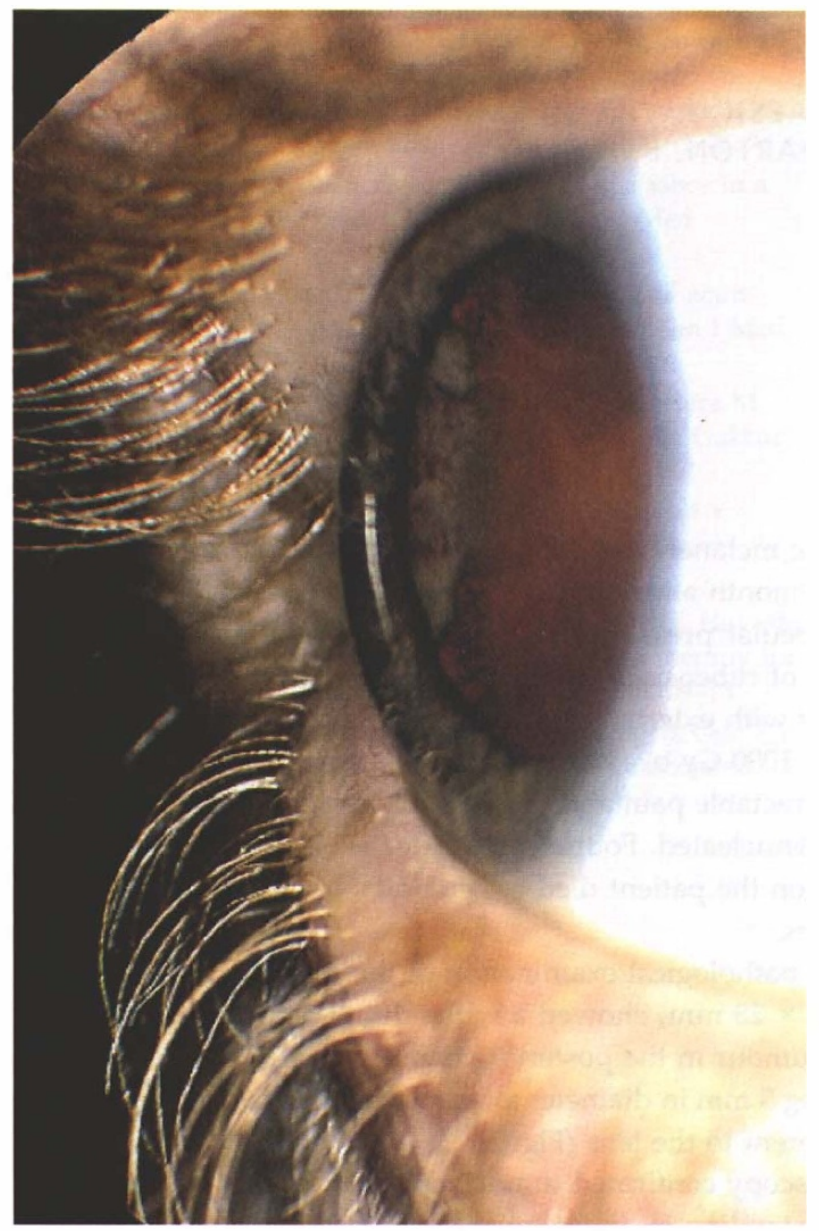

Fig. 1. Anterior segment photograph of the eye showing the tumour behind the iris.

iritis, pain and proptosis. Nevertheless metastatic cutaneous melanoma to the eye may remain asymptomatic. $^{3}$

Visual loss and blurring may be attributed to media opacity. ${ }^{4,5}$ Pigment dispersion in the aqueous, anterior vitreous and deposition over the lens surface contribute to media opacity. ${ }^{4,6}$ In addition iris nodules, prominent sentinel vessels, heterochromia iridis, hyphaema, iritis and secondary glaucoma have been described. $5,7-9$

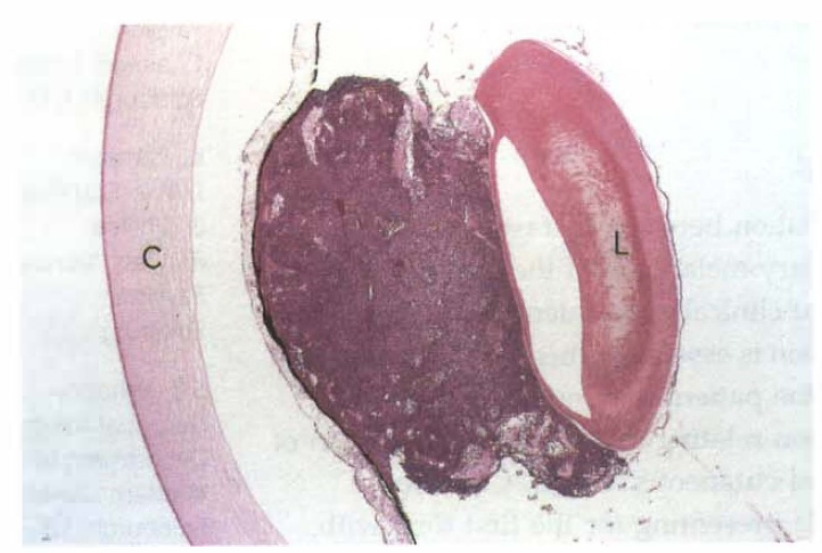

Fig. 2. Low-power view demonstrating a deposit of metastatic melanoma within the posterior chamber attached to the iris anteriorly and the surface of the lens posteriorly. C, cornea; $L$, lens $(H \mathcal{E E}, \times 20)$.
Tumour necrosis in the anterior segment may give rise to a brown or black hypopyon with similarly pigmented keratic precipitates. ${ }^{7,10,11}$ Necrosis may result from spontaneous regression, chemotherapy or external beam radiation. ${ }^{7,10,11}$

When anterior segment involvement predominates there is a tendency for diffuse scattering of isolated tumour cells in the anterior and posterior chamber. ${ }^{2}$ Elevated intraocular pressure may be seen at presentation or may develop during subsequent followup. Secondary open angle glaucoma may be due to direct angle invasion, obstruction of the trabecular meshwork by tumour cells or melanin-laden macrophages and inflammatory cells. ${ }^{4,5,12}$ Neovascular glaucoma may reflect a response to tumour angiogenesis factor; secondary angle closure glaucoma may also develop due to a mass effect. ${ }^{8}$

Ciliary body metastases have been shown histologically to invade the potential space between the non-pigmented and pigmented ciliary epithelium producing multiple elevated detachments of this layer that appear as nodules on the surface of ciliary body. ${ }^{2}$

Floaters with normal visual acuity may be the initial symptom in vitreous and retinal metastasis., ${ }^{73,14}$ In the case we report a history of floaters was elicited. Similar cases show golden-brown vitreous cells and spherules, vitreous strands without any discrete tumour mass, vitreous plaques, and clusters of golden-brown cells emanating from the region of the optic disc. ${ }^{7}$ Diffuse leakage of fluorescein in the peripapillary region was observed corresponding to the tumour deposits. Other posterior segment features described are pre-retinal, subretinal and vitreous haemorrhage. ${ }^{6,10}$ Histological features of retinal involvement include focal areas of cellular infiltration corresponding clinically to feathery periarteriolar retinal nerve fibre infiltrates. ${ }^{3}$ Cells disseminated throughout the vitreous have been found to arise from the retinal circulation, and to have scanty cytoplasm and pleomorphic hyperchromatic nuclei. ${ }^{4,13}$ Small tumour emboli were also found in the retinal vessels. $^{2,5,14}$

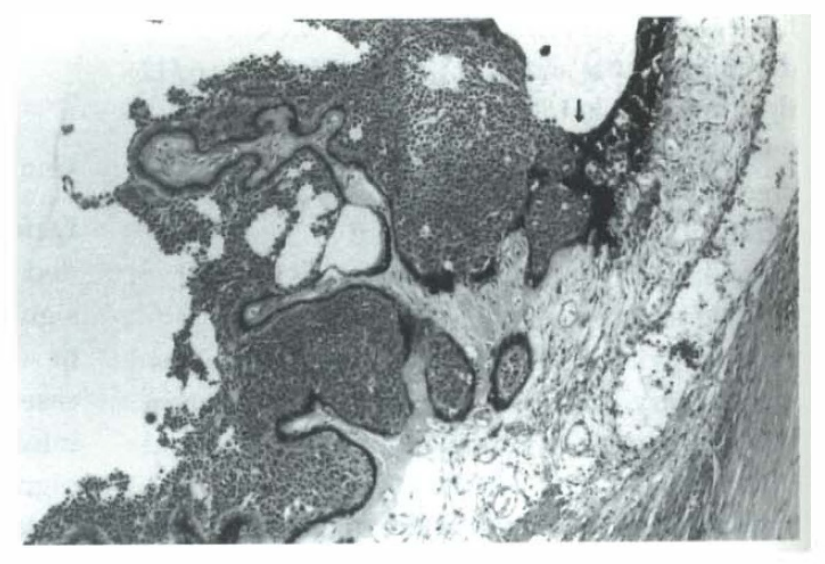

Fig. 3. Medium-power view showing tumour cells covering the surface of the ciliary body and within the anterior chamber angle (arrow) (HEE, $\times 100)$. 
Median age at presentation:

Time interval between diagnosis of skin primary and ocular metastasis:

Survival after ocular metastasis:

Number of identified locations:

Uveal

Choroid

Iris-ciliary body

Vitreo-retinal

Anterior chamber
46 years

22 months (18-56 months)

9 months (1-15 months)

79

$32(40 \%)$

$28(35 \%)$

$39(49 \%)$

$11(13 \%)$
Choroidal tumour mass may encroach on the posterior pole causing visual field defect. ${ }^{3,8,15}$ Choroidal involvement is, however, seldom solitary and has been shown histologically to be multifocal with evidence of tumour emboli within the choroidal vessels. ${ }^{4,8,15,16}$

Microscopic examination of the rare solitary metatases reveals similarities to the primary cutaneous biopsy specimen. This was confirmed by positive immunocytohistochemical staining for S100, neuronspecific enolase and HMB-45. ${ }^{8}$ Papilloedema and proptosis secondary to intracranial and orbital metastases may be encountered. ${ }^{13}$ Invasion of the optic nerve, meninges and orbit has been documented.

Histologically the majority of metastases are of the epithelioid cell type. ${ }^{2,8,17}$ Only two of the 27

histologically proven cases, including the case we report, are of the spindle cell variety. Aspiration cytology can be used to diagnose aqueous and vitreous involvement. ${ }^{2,7,14}$ Cytological smears stained with Papanicolaou stain may show features suggestive of malignancy.

The incidence of ocular metastasis of cutaneous melanoma is rare and has not been determined accurately. Shields et al. ${ }^{18}$ reported 40 cases of metastatic tumour to the iris, $3(8 \%)$ of which were from cutaneous melanoma. However, in combined data from three series only $1 \%$ of ocular metastases were from cutaneous melanoma. ${ }^{19}$ Gupta and Brasfield ${ }^{20}$ reported the incidence to be only $0.46 \%$, but of 15 consecutive patients who died of metastatic cutaneous malignant melanoma, $33 \%$ were found to have intraocular metastases at autopsy. ${ }^{17}$ Primary choroidal melanoma metastasising to cutaneous tissue is not infrequent and $\mathrm{Char}^{21}$ reported an incidence of $36 \%$ after a median follow-up of 4 years.

From the literature we identified 79 locations in 68 eyes. Fifty (75\%) uveal, 39 (49\%) vitreo-retinal and 11 $(13 \%)$ anterior chamber locations were identified. These figures show that uveal metastasis is commoner than metastasis to retino-vitreal locations (Table 1).

Differentiating features of primary uveal melanomas and metastatic cutaneous melanoma have been described. A past history of cutaneous malignancy and aggressive growth favour the diagnosis of metastasis. Primary iris melanomas tend to be of low-grade spindlecell type and may extend circumferentially. The tumour we report was of high grade, attached to, but not within the iris and spread as a sheet over the ciliary body, raising questions as to the site of entry into the eye. Primary choroidal melanomas are bulky, solitary, and consist of mixed spindle and epithelioid cells following
Callender's classification. ${ }^{2}$ Secondary melanomatous deposits in the choroid tend to be flat and multifocal, are epitheloid and evolve from tumour emboli. ${ }^{2,15}$

Bilateral primary malignant melanoma is virtually an unknown entity. ${ }^{22}$ Special characteristics of vitreo-retinal metastases from skin melanoma are golden-brown cells in the vitreous, vitreous plaques and clusters of vitreous cells on the retinal surface without discrete tumour mass. ${ }^{7}$ Anterior segment features suggestive of metastasis are pigment dispersion in the aqueous, black hypopyon and deposition over the lens.

Treatment of metastatic cutaneous melanoma to the eye has not been well defined. Therapeutic regimens to date have had varied success in inducing ocular tumour regression, although some treatment modalities have been unsuccessful. Therapeutic measures that have been advocated are enucleation, ${ }^{4,8,23}$ external beam radiation, ${ }^{14}$ chemotherapy, ${ }^{7,15}$ transfer factor, interferon and BCG vaccine. ${ }^{9}$ Intractable pain due to rubeotic glaucoma in a blind eye is considered to be an indication for enucleation. $4,8,23$

In the case we report tumour regression failed to occur with external beam radiation; similarly others found combined external beam radiation and intravenous vindesine failed to induce tumour regression. $^{8}$

Various chemotherapeutic agents have been used. Intravenous dicarbamazine, hydroxyurea and BCG vaccination resulted in intraocular tumour regression but failed to induce systemic regression. ${ }^{9}$ A bilateral metastatic uveal tumour from cutaneous melanoma was successfully managed with systemic BCNU (1,3-bis(2chloroethyl)-1-nitrosuria) in one case. ${ }^{24}$ However, combined administration of repeated systemic and subconjunctival DTIC (5-(3,3-dimethyl-1-

triazene)imidazol-4-carboxamide) and $\mathrm{BCNU}$ were unsuccessful in promoting tumour regression. ${ }^{13}$ It is interesting to note spontaneous regression has been reported in an eye with the development of black hypopyon. ${ }^{10}$

The majority of the patients at the time of ocular presentation had secondary deposits to regional lymph nodes, the central nervous system and viscera including lung, liver, gastrointestinal tract, pancreas, adrenal, heart, kidney, thyroid and spleen. ${ }^{20}$ The primary tumour metastasising to the eye.is predominantly of the superficial spreading variety; ${ }^{17}$ however, the nodular variety, which has the worst systemic prognosis, has also been reported. ${ }^{8}$ 
The time interval between diagnosis of skin primary and ocular manifestation varied from 18 to 56 months. ${ }^{7}$ Prognosis for survival varied similarly between 1 and 15 months.

In summary, this is a case of symptomatic metastatic melanoma to the posterior chamber attached to the anterior uveal tract and lens from a cutaneous primary, presenting with features of blurred vision, rubeosis iridis and later developing rubeotic glaucoma. Metastatic cutaneous malignant melanoma to the uveal tract is uncommon and involvement of the iris is rarer still. As the therapeutic measures for cutaneous melanoma improve, ocular metastasis may become a more common clinical problem.

\section{References}

1. de Bustros S, Augsburger JJ, Shields JA, Shakin EP, Pryor CC. Intraocular metastases from cutaneous malignant melanoma. Arch Ophthalmol 1985;103:937-40.

2. Font RL, Naumann G, Zimmerman LE. Primary malignant melanoma of the skin metastatic to the eye and orbit: report of ten cases and review of the literature. Am J Ophthalmol 1967;63:738-54.

3. Letson AD, Davidorf FH. Bilateral retinal metastases from cutaneous malignant melanoma. Arch Ophthalmol 1982;100:605-7.

4. Bowman CB, Guber D, Brown CH, Curtain VT. Cutaneous malignant melanoma with diffuse intraocular metastases. Arch Ophthalmol 1994;112:1213-5.

5. Cole EL, Zakov ZN, Meisler DM, Tuthill RJ, McMahon JT. Cutaneous malignant melanoma metastatic to the vitreous Arch Ophthalmol 1986;104:98-101.

6. Spraul CW, Martain DF, Hagler WS, Grossiniklaus HE. Cytology of metastatic cutaneous melanoma to the vitreous and retina. Retina 1996;16:328-32.

7. Char DH, Schwartz A, Miller TR, Abele JS. Ocular metastases from systemic melanoma. Am J Ophthalmol 1980;90:702-7.

8. Bell RWD, Ironside JW, Fleck BW, Singh J. Cutaneous malignant melanoma metastatic to the choroid: a clinicopathological case report. Eye 1995;9:650-3.

9. Hirst LW, Reich J, Galbraith JEK. Primary cutaneous malignant melanoma metastatic to the iris. $\mathrm{Br} \mathrm{J}$ Ophthalmol 1979;63:165-8.
10. Wormald RPL, Harper JI. Bilateral black hypopyon in a patient with self-healing cutaneous malignant melanoma. $\mathrm{Br}$ J Ophthalmol 1983;67:321-35.

11. Albert DM, Lahav M, Trocznski E, Bahr R. Black hypopyon: report of two cases. Graefes Arch Klin Ophthalmol 1975;193:81-94.

12. Yanoff M, Scheie HG. Melanomalitic glaucoma: report of a case. Arch Ophthalmol 1970;84:471-3.

13. Robertson DM, Wilkinson CP, Murray JL, Gordy DD. Metastatic tumour to the retina and vitreous cavity from primary melanoma of the skin. Ophthalmology 1981;88:1296-301.

14. Gunduz K, Shields JA, Shields CL, Eagle RC. Cutaneous melanoma metastatic to the vitreous cavity. Ophthalmology 1998;105:600-5.

15. Greven CM, Slusher MM, Stanton C, Yeatts P. Cutaneous malignant melanoma metastatic to the choroid. Arch Ophthalmol 1991;109:547-9.

16. Covel LL, Markiewitz HH. The choroid as the primary manifestation of systemic involvement in skin melanoma: report of a case. Am J Ophthalmol 1961;51:1296-303.

17. Fishman ML, Tomaszewski MM, Kuwabara T. Malignant melanoma of the skin metastatic to the eye. Arch Ophthalmol 1976;94:1309-11.

18. Shields JA, Shields CL, Kiratli H, De Potter P. Metastatic tumours to the iris in 40 patients. Am J Ophthalmol 1995;119:422-30.

19. McLean IW, Burnier MN, Zimmerman LE, Jakobiec FA. Tumours of the uveal tract. In: Atlas of tumour pathology: tumors of the eye and ocular adnexa. 3rd series. Washington, DC: AFIP, 1994:chap. 5.

20. Gupta TD, Brasfield R. Metastatic melanoma. Cancer 1964;17:1323-39.

21. Char DH. Metastatic choroidal melanoma. Am J Ophthalmol 1978;86:76-80.

22. Holck DEE, Dutton JJ, Pendergast SD, Gordon KK. Double choroidal malignant melanoma in an eye with apparent clinical regression. Surv Ophthalmol 1998;42:393-416.

23. Riffenburgh RS. Malignant melanoma to the retina. Arch Ophthalmol 1962;66:487-9.

24. Stark WJ, Rosenthal ARE, Mullins GM, Green WR. Simultaneous bilateral uveal melanomas responding to BCNU therapy. Trans Am Acad Ophthalmol Otolaryngol 1971;75:70-83. 